\title{
Puerarin exhibits antiinflammatory properties in gunpowder smog-induced acute lung injury in rats via regulation of the renin-angiotensin system and the NFKB signaling pathway
}

\author{
FAN ZHANG，YU WANG, PEIPEI LIU, PING DI, MIANYANG LI and CHENGBIN WANG \\ Department of Clinical Laboratory Medicine, Chinese People's Liberation Army General Hospital \\ and Postgraduate Medical School, Beijing 100853, P.R. China
}

Received October 29, 2020; Accepted April 28, 2021

DOI: $10.3892 / \mathrm{etm} .2021 .10241$

\begin{abstract}
Puerarin, which is a widely used in Traditional Chinese Medicine, was previously demonstrated to regulate the subsets of $\mathrm{CD}^{+}$lymphocytes in gunpowder smog-induced acute lung injury (ALI). However, the underlying mechanism remains largely unknown. Previous studies on autoimmune diseases have revealed that the renin-angiotensin system (RAS) and NF- $\kappa \mathrm{B}$ participate in regulating the levels of $\mathrm{CD}^{+}$ $\mathrm{T}$ lymphocytes. The aim of the present study was to further investigate the mechanisms underlying the protective effects of puerarin. Wistar rats were randomly divided into four groups as follows: Normal control, puerarin control, smoke inhalation injury and puerarin treatment plus smoke inhalation injury groups. The levels of angiotensin II (Ang II) in lung tissue and in the circulation, and the levels of interleukin (IL)-6, IL-1 $\beta$, IL-17A and tumor necrosis factor (TNF)- $\alpha$ in the bronchoalveolar lavage fluid (BALF) were assayed using ELISA kits. The expression of Ang II type 1 receptor (AT1-R), angiotensin-converting enzyme (ACE) and ACE2 were examined by immunohistochemical analysis and western blotting. Phosphorylated (p-) NF- $\mathrm{B}$ p65 and NF- $\kappa \mathrm{B}$ inhibitor $\alpha$ $(\mathrm{I} \kappa \mathrm{B}-\alpha)$ protein expression levels were also determined using western blotting. Puerarin treatment reduced the levels of inflammatory cytokines in the BALF. Furthermore, puerarin treatment significantly decreased the levels of Ang II, AT1-R and ACE, which were increased following smoke inhalation. Conversely, puerarin treatment upregulated the expression of ACE2, which was downregulated following smoke inhalation. Additionally, puerarin decreased the expression of $\mathrm{p}-\mathrm{NF}-\kappa \mathrm{B}$
\end{abstract}

Correspondence to: Dr Chengbin Wang or Dr Mianyang Li, Department of Clinical Laboratory Medicine, Chinese People's Liberation Army General Hospital and Postgraduate Medical School, 28 Fuxing Road, Beijing 100853, P.R. China

E-mail: sysuveronica@126.com

E-mail: 745532256@qq.com

Key words: smoke inhalation injury, puerarin, renin-angiotensin system, nuclear factor- $\mathrm{kB}$ p65 and increased that of IkB- $\alpha$. Thus, the antiinflammatory effects of puerarin were partly mediated via the RAS and via regulation of the NFKB signaling pathway in rats with gunpowder smog-induced ALI.

\section{Introduction}

Smoke inhalation injury is one of the primary causes of acute lung injury (ALI) or acute respiratory distress syndrome (ARDS), affecting $>50,000$ individuals annually $(1,2)$. In our previous study, it was demonstrated that subsets of $\mathrm{CD}^{+} \mathrm{T}$ lymphocytes, including regulatory $\mathrm{T}$ cells (Tregs) and T-helper (Th) 17 cells, served critical roles in the development of smoke inhalation-induced ALI (3). Puerarin, a compound used widely in Traditional Chinese Medicine, was found to exert protective effects against gunpowder smog-induced ALI by inhibiting Th17 responses (4). However, the molecular mechanisms by which puerarin exerted its effects require further study.

Angiotensin(Ang) II is a key memberof the renin-angiotensin system (RAS), and it is involved in the regulation of inflammation, proliferation and fibrosis in ALI (5). Notably, research on autoimmune diseases has demonstrated that Ang II and its related signaling pathways, such as the NF- $\kappa \mathrm{B}$ pathway, may be involved in the regulation of $\mathrm{CD}^{+} \mathrm{T}$ lymphocytes. Blocking the interaction of Ang II with Ang II type 1 receptor (AT1-R) resulted in upregulation of antigen-specific Tregs and inhibited autoreactive Th17 cells (6). A recent study on colonic inflammation demonstrated that the local RAS in the colon is activated in colitis and promotes colonic inflammation by inducing Th17-cell activation (7). Ang II can induce the secretion of interleukin (IL)-6, which together with TGF- $\beta$ can then promote the differentiation of Th17 cells $(8,9)$.

It has been demonstrated that puerarin suppressed murine hemangioendothelioma cell proliferation during Ang II-induced aortic aneurysm formation by affecting the rate of apoptosis (10). In addition, a previous study has shown that puerarin attenuated Ang II-induced cardiac fibroblast proliferation by increasing catalase activity and inhibiting hydrogen peroxide-dependent Rac-1 activation (11).

As it was demonstrated that puerarin may attenuate Ang II-induced inflammation in other diseases, the present 
study was undertaken to determine whether puerarin may alleviate inflammation via the RAS and the NF- $\kappa \mathrm{B}$ pathway in gunpowder smog-induced ALI.

\section{Materials and methods}

Animals and reagents. Male Wistar rats, aged 8-10 weeks and weighing 290-310 g, were supplied by the Laboratory Animal Center of the People's Liberation Army GeneralHospital (Beijing, China), and raised in accordance with the National Institutes of Health on Animal Care and Ethical Guidelines (4). Rats were raised in pathogen-free cages, maintained at a temperature of $20-25^{\circ} \mathrm{C}$ and a relative humidity of $50-70 \%$, and received food and water ad libitum. All experimental procedures were approved by the Ethics Committee of the Medical College of the People's Liberation Army [approval no. SCXK (Beijing) 2012-0001]. Puerarin $(50 \mathrm{mg} / \mathrm{ml})$ was obtained from Chengdu Tiantaishan Pharmaceutical Co., Ltd. (4).

Animal model and experimental procedures. The rat model was established based on our previous study (3). Using a self-made smoke generator, smoke was generated from $10 \mathrm{~g}$ gunpowder. The electromagnetic heater was turned off as soon as the flame burnt out. A total of two rats at a time were exposed to the smoke for $8 \mathrm{~min}(3)$. The situation of rats was monitored every $2 \mathrm{~min}$.

A total of 40 rats were randomly divided into four groups as follows: Control group (termed Con; saline administration plus ambient air inhalation), puerarin control group (termed Pue; puerarin administration plus ambient air inhalation), ALI group (termed ALI; saline administration plus smoke inhalation), and the puerarin treatment group (termed ALI + Pue; puerarin administration plus smoke inhalation). The rats in the Con and ALI groups were administered with normal saline $(\mathrm{NaCl}, 0.9 \%)$ intraperitoneally. The rats in the ALI + Pue group were injected with puerarin intraperitoneally (100 mg/kg) $30 \mathrm{~min}$ after smoke inhalation. In the Pue group, puerarin was administered intraperitoneally $(100 \mathrm{mg} / \mathrm{kg})$ without smoke inhalation. The dose of puerarin used was based on a previous study (4). A total of $24 \mathrm{~h}$ after smoke exposure, all the rats were anesthetized with sodium pentobarbital intraperitoneally (50 mg/kg; Sigma-Aldrich; Merck KGaA), dissolved in normal saline at a concentration of $10 \mathrm{mg} / \mathrm{ml}$. Specific criteria (humane endpoints) were used to determine when rats should be euthanized. While under anesthesia, all the rats were euthanized by exsanguination: $10 \mathrm{ml}$ blood was collected from the abdominal aorta of the rats (weighing 290-310 g) for further analysis. Death was confirmed based on the arrest of the cardiac and respiratory function ( $\geq 5 \mathrm{~min})$. After sacrifice, the left lung was prepared for collection of bronchoalveolar lavage fluid (BALF). The right upper lobe of the lung was used for immunohistochemistry analysis and the right lower lobe of the lung was stored at $-80^{\circ} \mathrm{C}$.

Measurement of arterial blood gases. Arterial blood was collected from the abdominal aorta using a $10 \mathrm{ml}$ syringe containing heparinized saline. The blood samples were immediately injected into an ABL700 blood gas analyzer (Radiometer) to measure the $\mathrm{pH}$ and the partial gas pressures of oxygen $\left(\mathrm{PaO}_{2}\right)$ and carbon dioxide $\left(\mathrm{PaCO}_{2}\right)$.
$B A L F$ analysis. The experimental protocols used were performed as described previously (4). The supernatant was prepared for the cytokine assays.

Ang II determination. The lung tissue samples (100 mg each) were homogenized in normal saline and centrifuged for $20 \mathrm{~min}$ at $10,000 \mathrm{x}$ g at $4^{\circ} \mathrm{C}$ (3). Blood samples were collected from the abdominal aorta, and serum was obtained by centrifugation (1,000 x $\mathrm{g}$ for $10 \mathrm{~min})$. The concentration of Ang II in the lung homogenates and serum were determined using ELISA kits, according to the manufacturer's protocol (cat. no. 1158/5; R\&D Systems, Inc.).

Cytokine assays. The concentrations of the proinflammatory cytokines IL-6, IL-1 $\beta$, IL-17A and TNF- $\alpha$ in the BALF were determined using ELISA kits (cat. nos. R6000B, RLB00, DY8410-05 and RTA00, respectively; R\&D Systems, Inc.), following the manufacturer's protocol. The absorbance was measured at $450 \mathrm{~nm}$ using a microplate reader (BioTek Instruments, Inc.).

Immunohistochemistry. The lung tissue was fixed with $4 \%$ paraformaldehyde (cat. no. P1110; Beijing Solarbio Science $\&$ Technology Co., Ltd.) for $12 \mathrm{~h}$ at $4^{\circ} \mathrm{C}$. Paraffin-embedded $5-\mu \mathrm{m}$ sections of lung tissues were deparaffinized in xylene and hydrated in a decreasing series of ethanol solutions. The sections were blocked using $10 \%$ normal goat serum (cat. no. SL038; Beijing Solarbio Science \& Technology Co., Ltd.) for $1 \mathrm{~h}$ at room temperature. The sections were probed with specific primary antibodies in a humidified chamber at $4^{\circ} \mathrm{C}$ overnight and secondary horseradish peroxidase (HRP)-conjugated antibodies at room temperature for $1 \mathrm{~h}$. The primary antibodies used were: Monoclonal mouse anti-angiotensin-converting enzyme (ACE; cat. no. ab270712; 1:100), rabbit monoclonal anti-ACE2 (cat. no. ab108252; 1:100) and rabbit polyclonal anti-AT1-R (cat. no. ab124505; 1:100) (all from Abcam). The secondary HRP-conjugated antibodies used were goat anti-mouse or anti-rabbit IgG (cat. nos. ZB-2305 and ZB-2301, respectively; 1:2,000; Zhongshan Bio-Tech Co, Ltd.). Specific staining was detected using the two-step streptavidin-peroxidase method (Non-Biotin HRP Direction System; Zhongshan Bio-Tech Co, Ltd.). The signals were developed using 3,3'-diaminobenzidine. Subsequently, the sections were counterstained using Mayer's hematoxylin, dehydrated, cleared in xylene and imaged using a light microscope (x400 magnification; Carl Zeiss AG).

Western blotting. Lung tissues were homogenized using liquid nitrogen. RIPA lysis buffer (Beyotime Institute of Biotechnology) containing PMSF (Beyotime Institute of Biotechnology) was used for the lysis of the cells, followed by centrifugation at $4^{\circ} \mathrm{C}(10,000 \mathrm{x} \mathrm{g}$ for $15 \mathrm{~min})$. Protein concentrations in lung tissues were determined using a BCA protein assay kit (Novagen; Merck KGaA). Protein samples $(50 \mu \mathrm{g})$ were loaded on a $10 \%$ SDS-gel, resolved using SDS-PAGE and transferred to a PVDF membrane. The membrane was blocked using 5\% skimmed milk in Tris-buffered saline (10 mM Tris, $150 \mathrm{mM} \mathrm{NaCl}$, pH7.4) with $0.1 \%$ Tween-20 (TBST) at room temperature for $1 \mathrm{~h}$. The membrane was probed using specific primary antibodies at $4^{\circ} \mathrm{C}$ 
A

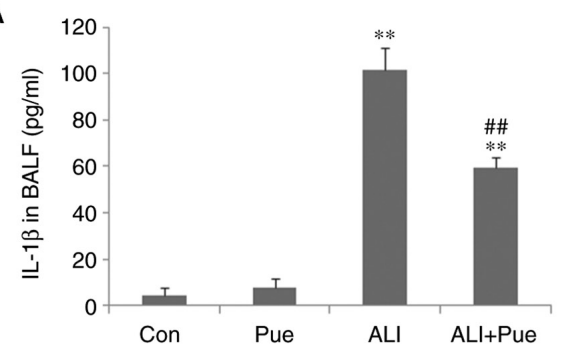

C

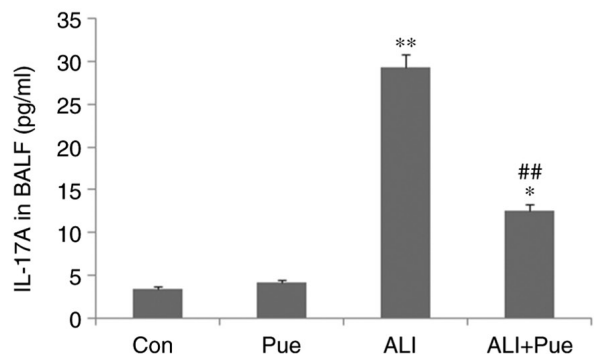

B

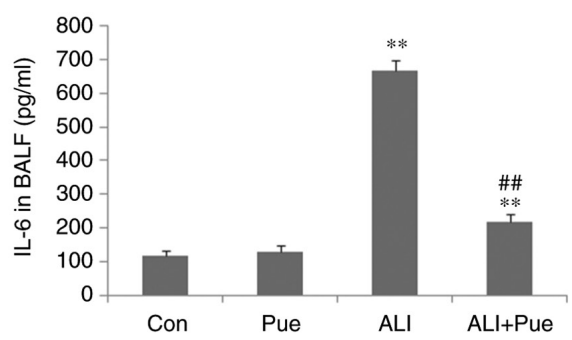

D

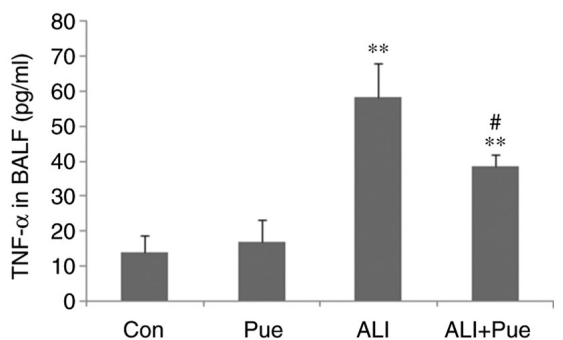

$\mathrm{E}$

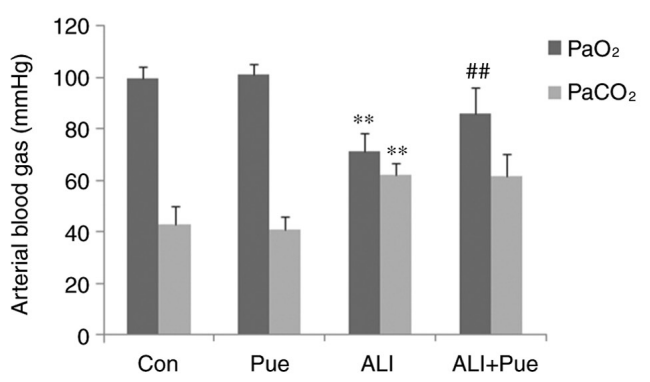

Figure 1. Cytokine levels in BALF and analysis of arterial blood gases. (A) IL-1 $\beta$, (B) IL-6, (C) IL-17A and (D) TNF- $\alpha$ concentrations in BALF were determined using ELISA. (E) Arterial blood gases, including $\mathrm{PaO}_{2}$ and $\mathrm{PaCO}_{2}$ were analyzed. Data are presented as mean \pm standard error of the mean from three independent experiments ( $\mathrm{n}=10$ per group). ${ }^{*} \mathrm{P}<0.05,{ }^{* *} \mathrm{P}<0.01$ vs. Con group; ${ }^{\#} \mathrm{P}<0.05$, ${ }^{\#} \mathrm{P}<0.01$ vs. ALI group. BALF, bronchoalveolar lavage fluid; $\mathrm{PaO}_{2}$, partial gas pressure of oxygen; $\mathrm{PaCO}_{2}$, partial gas pressure of carbon dioxide; Con, normal control group; Pue, puerarin control group; ALI, smoke inhalation group; ALI + Pue, puerarin treatment plus smoke inhalation group.

overnight and secondary HRP-conjugated antibodies at room temperature for $1 \mathrm{~h}$. The primary antibodies used were: Mouse monoclonal anti-ACE (cat. no. ab270712; 1:100; Abcam), rabbit monoclonal anti-ACE2 (cat. no. ab108252; 1:1,000; Abcam), rabbit polyclonal anti-AT1-R (cat. no. ab124505; 1:800; Abcam) anti-GAPDH (cat. no. ab9485; 1:1,000; Abcam), mouse monoclonal anti-NF-кB p65 (cat. no. 6956; 1:1,000; Cell Signaling Technology, Inc.), mouse monoclonal anti-phosphorylated (p-) NF-кB p65 (cat. no. 13346; 1:1,000; Cell Signaling Technology, Inc.) and mouse monoclonal

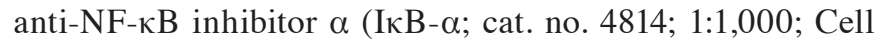
Signaling Technology, Inc.). The secondary HRP-conjugated antibodies used were goat anti-mouse and anti-rabbit IgG (cat. nos. PV9002 and PV9001, respectively; 1:2,000; Zhongshan Bio-Tech Co, Ltd.). After washing with TBST, the signals were developed using an enhanced chemiluminescence detection kit (cat. no. P0018S; Beyotime Institute of Biotechnology), imaged using a GelDoc XR automated gel imaging system (Bio-Rad Laboratories, Inc.) and analyzed using Image Lab version 3.0 (Bio-Rad Laboratories, Inc.).

Statistical analysis. All data are presented as the mean \pm standard error mean of at least three independent experiments. Differences in the means of the groups were compared using the Bonferroni post hoc test following Kruskal-Wallis in SPSS version 17.0 (SPSS Inc.). Dunn's test was used for comparing two groups. $\mathrm{P}<0.05$ was considered to indicate a statistically significant difference.

\section{Results}

Analysis of arterial blood gases. Following smoke inhalation, arterial blood gases, including $\mathrm{PaO}_{2}$ and $\mathrm{PaCO}_{2}$ were significantly altered (Fig. 1E). Hypoxemia occurred during the early stage of ALI/ARDS. A total of $24 \mathrm{~h}$ after smoke exposure, the rats developed hypoxemia and hypercapnia with decreased $\mathrm{PaO}_{2}$ and increased $\mathrm{PaCO}_{2}$ compared with the control group (Fig. 1E). Puerarin administration improved the hypoxemic symptoms by increasing $\mathrm{PaO}_{2}$ (Fig. 1E). However, the $\mathrm{PaCO}_{2}$ was not significantly decreased in the puerarin treatment group compared with the ALI group (Fig. 1E).

Proinflammatory cytokine levels in the BALF. As shown in Fig. 1A-D, the levels of IL-6, IL-1 $\beta$, IL-17A and TNF- $\alpha$ in the BALF in the control groups were low. The levels of IL-6, IL-1 $\beta$, IL-17A and TNF- $\alpha$ in the BALF significantly increased following exposure to smoke (Fig. 1A-D). Puerarin treatment significantly reduced the levels of IL-6, IL-1 $\beta$, IL-17A and TNF- $\alpha$ compared with the ALI group, particularly those of IL-6 and IL-17A (Fig. 1A-D). This suggests that puerarin may reduce Th17 cell levels by decreasing the levels of related cytokines, including IL-6 and IL-17A. The concentration of IL-6, IL-1 $\beta$, 

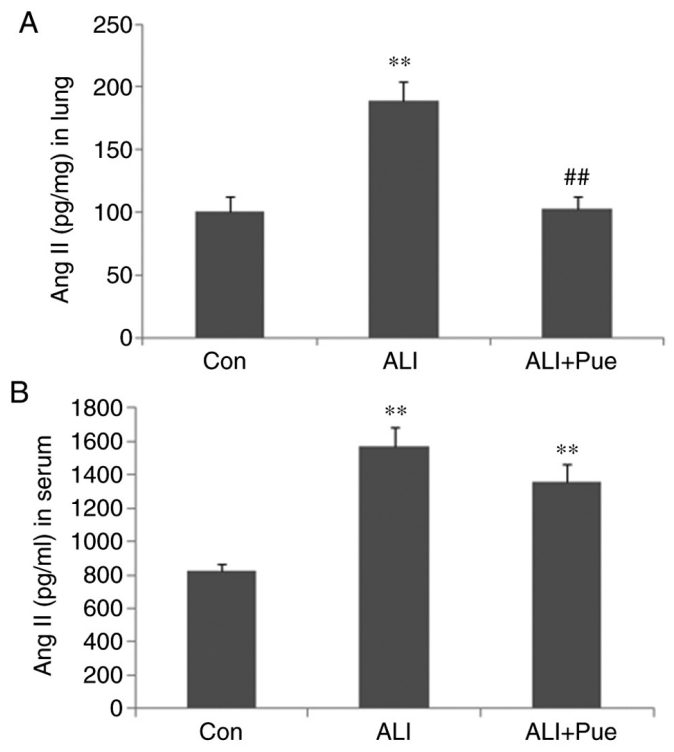

Figure 2. Determination of Ang II levels. The concentrations of Ang II in (A) lung homogenates and (B) serum were determined using ELISA. Data are presented as mean \pm standard error of the mean from three independent experiments ( $\mathrm{n}=10$ per group). ${ }^{* *} \mathrm{P}<0.01$ vs. Con group; ${ }^{\# \#} \mathrm{P}<0.01$ vs. ALI group. Ang II, angiotensin II; Con, normal control group; ALI, smoke inhalation group; ALI + Pue, puerarin treatment plus smoke inhalation group.

IL-17A and TNF- $\alpha$ in the BALF was not significantly different between the Con and Pue groups (Fig. 1A-D).

Ang II levels in the lung tissues and circulation. To assess the effects of puerarin on Ang II production, the Ang II levels in the lungs and circulation were assayed. As shown in Fig. 2, the levels of Ang II were significantly increased following smoke inhalation in both the lung tissues and the peripheral blood of rats. Puerarin treatment significantly reduced Ang II levels in the lung tissues (Fig. 2A), but not in the serum (Fig. 2B). Thus, the suppression of activation of the local RAS in the lung may be more important for the protective effects of puerarin, compared with the RAS in the peripheral blood.

Effect of puerarin on the expression of AT1-R. Immunohistochemical staining was used to determine the localization of AT1-R in the lung tissue of rats. AT1-R was expressed abundantly in inflammatory cells in the alveoli and interstitial tissue (Fig. 3A-C).

The protein expression levels of AT1-R in the lung tissues were quantified using western blotting. Fig. 3D shows representative images of the immunoreactive bands $(\sim 45 \mathrm{kDa}$ in weight). The protein expression levels of AT1-R in the lungs were significantly increased $24 \mathrm{~h}$ following smoke inhalation compared with those in the control group (Fig. 3D and E). Of note, AT1-R protein expression levels were decreased in the lungs following treatment with puerarin compared with those in the ALI group (Fig. 3D and E).

Effect of puerarin on the expression of ACE. Immunohistochemical staining was used to determine the localization of ACE in the lung tissues of rats. ACE expression was evident in alveolar epithelial cells, as well as in endothelial cells (Fig. 4A-C).
ACE expression in the lungs was quantified using western blotting, and Fig. 4D shows representative images of the immunoreactive bands $(\sim 195 \mathrm{kDa})$. The protein expression levels of ACE in the lungs increased $24 \mathrm{~h}$ following smoke inhalation compared with those in the control group (Fig. 4D and E). Notably, ACE levels significantly decreased in the lungs following puerarin administration compared with those in the lungs after smoke inhalation alone (Fig. 4D and E).

Effect of puerarin on the expression of ACE2. Immuno-histochemical staining was used to determine the localization of ACE2 in the lung tissues of rats. The major sites of ACE2 expression were the alveolar epithelial and endothelial cells (Fig. 5A-C).

The levels of ACE2 in the lungs were quantified using western blotting, and Fig. 5D shows representative images of the immunoreactive bands $(\sim 90 \mathrm{kDa})$. The protein expression levels of ACE2 in the lungs decreased following smoke inhalation compared with those in the control group (Fig. 5D and E). Furthermore, the protein expression levels of ACE2 increased in the lungs following administration of puerarin compared with those in the lungs after smoke inhalation (Fig. 5D and E).

Effect of puerarin on the expression of $p-N F-\kappa B$ p 65 and $I \kappa B-\alpha$. The phosphorylation status of $\mathrm{NF}-\kappa \mathrm{B}$ p 65 and the expression levels of $\mathrm{I} \kappa \mathrm{B}-\alpha$ in the lungs were quantified using western blotting. The results revealed that the expression ratio of $\mathrm{p}-\mathrm{NF}-\kappa \mathrm{B}$ p 65 relative to total $\mathrm{NF}-\kappa \mathrm{B}$ p65 in the lungs was significantly increased $24 \mathrm{~h}$ following smoke inhalation compared with that in the control group (Fig. 6A). Notably, $\mathrm{NF}-\kappa \mathrm{B}$ p 65 phosphorylation was decreased in the lungs following puerarin treatment compared with the ALI group (Fig. 6A).

The protein expression levels of I $\mathrm{B}-\alpha$ in the lungs decreased after smoke inhalation compared with those in the control group (Fig. 6B). In addition, I $\mathrm{B}-\alpha$ protein expression levels increased in the lungs following administration of puerarin compared with that in the lungs after smoke inhalation (Fig. 6B). Thus, puerarin treatment inhibited the activation of the NF- $\kappa \mathrm{B}$ pathway by downregulating the phosphorylation of $\mathrm{NF}-\kappa \mathrm{B}$ p65 and upregulating $\mathrm{I} \kappa \mathrm{B}-\alpha$ expression.

\section{Discussion}

In our previous study, it was demonstrated that puerarin attenuated inflammatory responses by inhibiting Th17 responses (4). However, the underlying mechanisms involved were not determined. In autoimmune diseases, it has been shown that the RAS and the NF- $\kappa \mathrm{B}$ pathway may contribute to the modulation of the levels of $\mathrm{CD}^{+} \mathrm{T}$ lymphocytes (6). The RAS is primarily composed of Ang II, AT1-R, ACE, ACE2 and Ang (1-7). ACE and ACE2 share homology in their catalytic domains but have different key functions in the RAS. ACE cleaves Ang I to generate Ang II, whereas ACE2 is a negative regulator of the system, both degrading the vasoconstrictor Ang II and producing the vasodilator Ang (1-7) (12).

A previous study explored the effects of a combination of felodipine + puerarin on the ACE2/Ang (1-7)/Mas axis, to investigate the protective effects of this combination against 


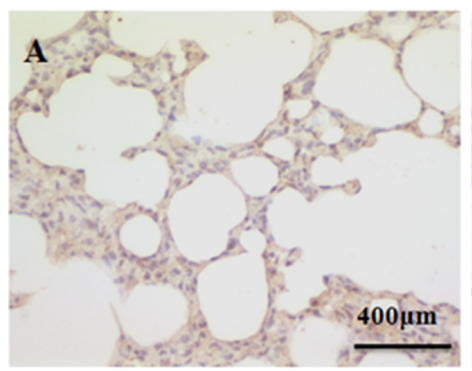

D

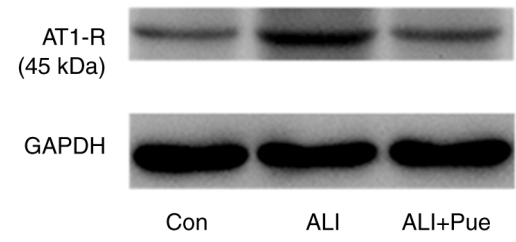

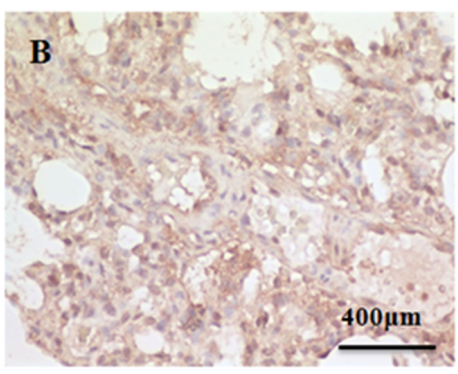
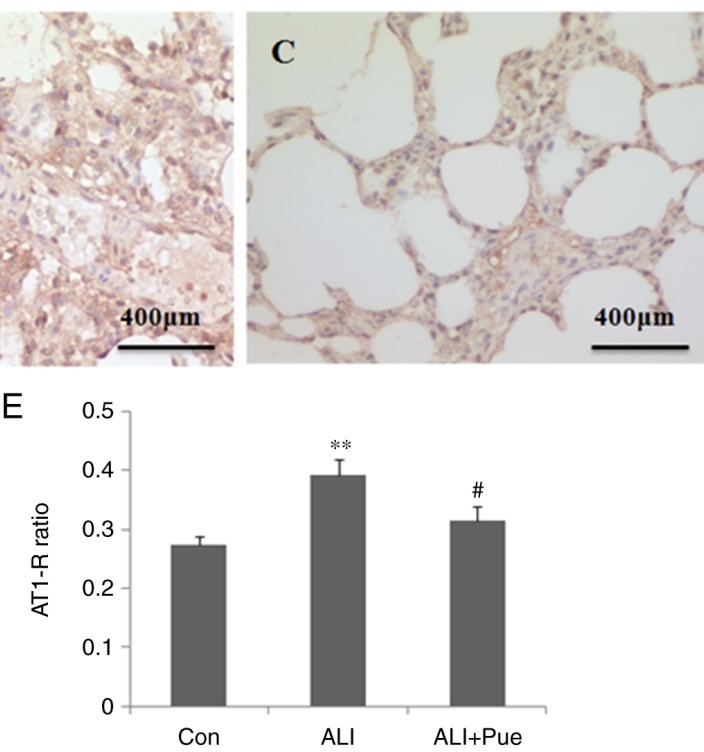

Figure 3. Effect of puerarin on AT1-R expression. (A) Representative images from immunohistochemical analysis of AT1-R expression in lung tissue sections from rats of the Con, (B) ALI and (C) ALI + Pue groups. (D) Representative images from western blot analysis for AT1-R protein expression levels in lung tissues. (E) Quantification of AT1-R protein expression levels relative to GAPDH. Data are presented as the mean \pm the standard error of the mean from three independent experiments ( $\mathrm{n}=10$ per group). ${ }^{* *} \mathrm{P}<0.01$ vs. Con group; ${ }^{*} \mathrm{P}<0.05$ vs. ALI group. AT1-R, angiotensin II type 1 receptor; Con, normal control group; ALI, smoke inhalation group; ALI + Pue, puerarin treatment plus smoke inhalation group.

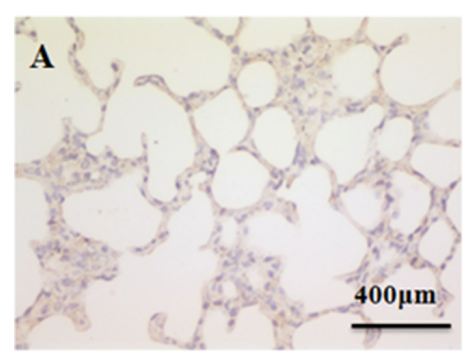

D

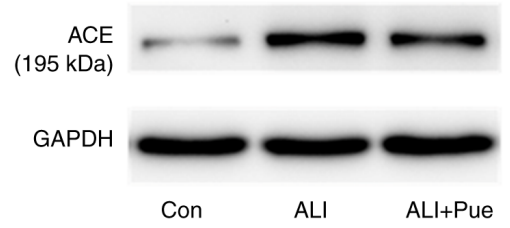

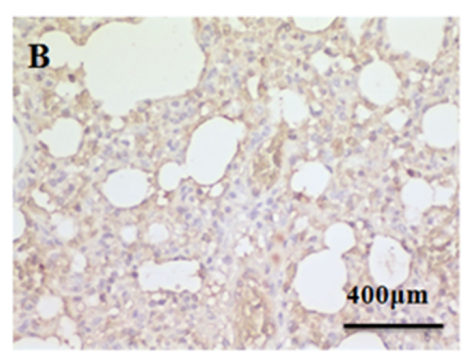

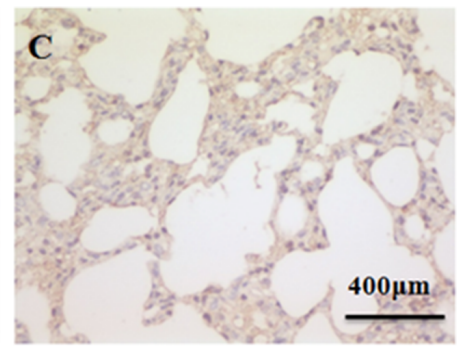

E

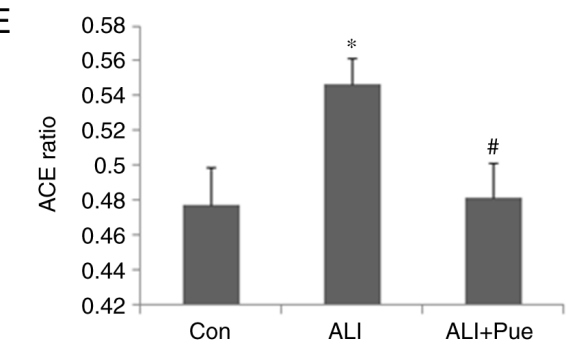

Figure 4. Effect of puerarin on ACE expression. (A) Representative images from immunohistochemical analysis of ACE expression in lung tissue sections from rats of the Con group, (B) ALI and (C) ALI + Pue groups. (D) Representative images from western blot analysis for ACE protein expression levels in the lungs. (E) Quantification of ACE protein expression levels relative to GAPDH. Data are presented as the mean \pm the standard error of the mean from three independent experiments ( $\mathrm{n}=10$ per group). ${ }^{*} \mathrm{P}<0.05$ vs. Con group; ${ }^{*} \mathrm{P}<0.05$ vs. ALI group. ACE, angiotensin-converting enzyme; Con, normal control group; ALI, smoke inhalation group; ALI + Pue, puerarin treatment plus smoke inhalation group.

kidney damage in renovascular hypertensive rats. Compared with the felodipine group, felodipine + puerarin significantly attenuated fibrosis, decreased Ang II levels and increased Ang (1-7) levels, upregulated the mRNA expression levels of ACE2 in the bilateral kidneys, as well the levels of Mas in the ischemic kidney, downregulated the levels of ACE in bilateral kidneys and that of AT1-R in the ischemic kidney, and significantly decreased the expression of TGF- $\beta$ (13). Therefore, it was hypothesized that puerarin may also exert its anti-inflammatory effects by regulating the RAS and NF- $\kappa$ B pathways in gunpowder smog-induced ALI.
In the present study, blood gas analysis demonstrated that puerarin could reverse hypoxemia post-smoke inhalation. However, levels of $\mathrm{PaCO}_{2}$ were unchanged by puerarin treatment. Further studies should expand the experimental scale to confirm the results and determine the underlying mechanisms involved. In addition, puerarin significantly reduced the levels of IL-6, IL-1 $\beta$, IL-17A and TNF- $\alpha$ in the BALF, suggesting that puerarin may attenuate cytokine-mediated inflammation. However, the underlying mechanisms remain to be determined.

The RAS is intricately involved in maintaining blood pressure homeostasis, as well as the balance of body 

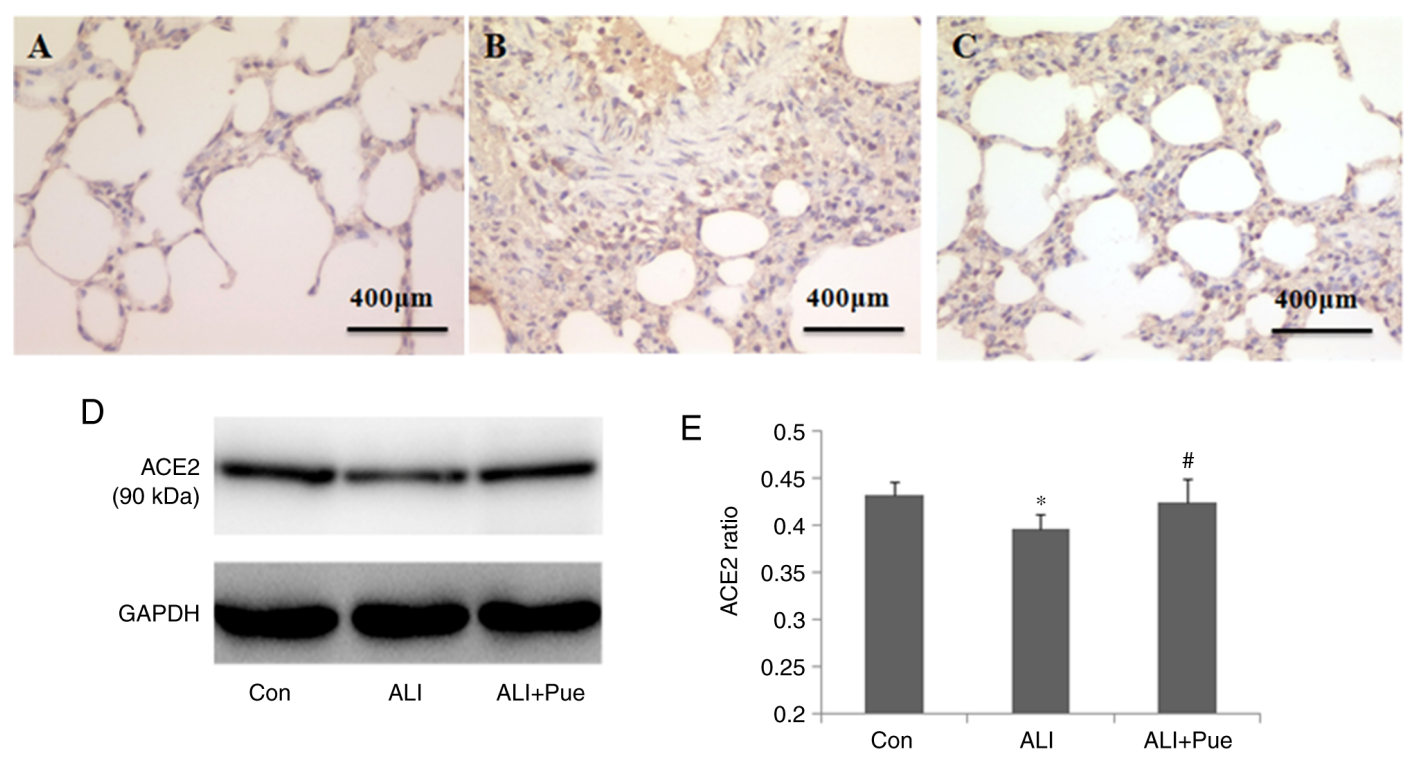

Figure 5. Effect of puerarin on ACE2 expression. (A) Representative images from immunohistochemical analysis of ACE2 expression in lung tissue sections from rats of the Con group, (B) ALI and (C) ALI + Pue groups. (D) Representative images from western blot analysis for ACE2 protein expression levels in the lungs. (E) Quantification of ACE protein expression levels relative to GAPDH. Data are presented as the mean \pm the standard error of the mean from three independent experiments ( $\mathrm{n}=10$ per group). ${ }^{*} \mathrm{P}<0.05$ vs. Con group; ${ }^{~} \mathrm{P}<0.05$ vs. ALI group. ACE2, angiotensin-converting enzyme 2 ; Con, normal control group; ALI, smoke inhalation group; ALI + Pue, puerarin treatment plus smoke inhalation group.

A

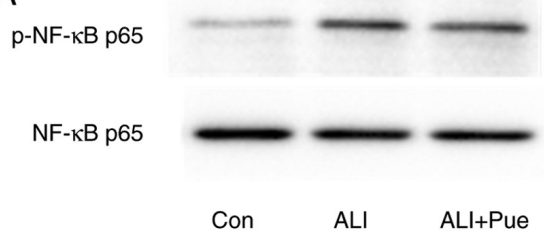

B

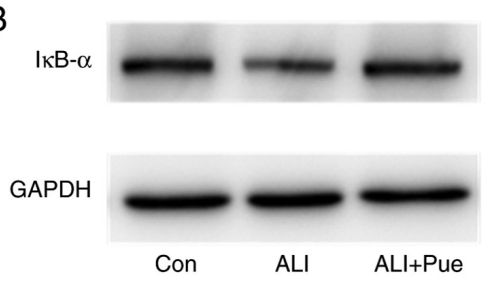

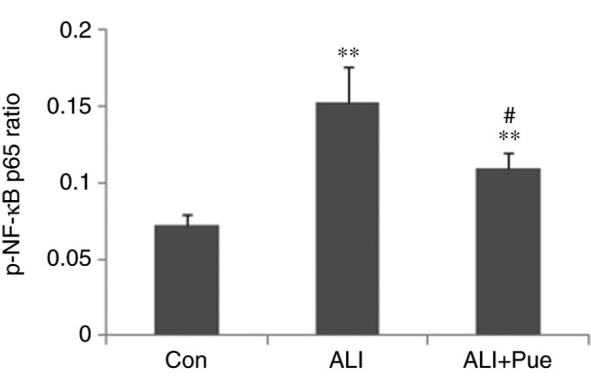

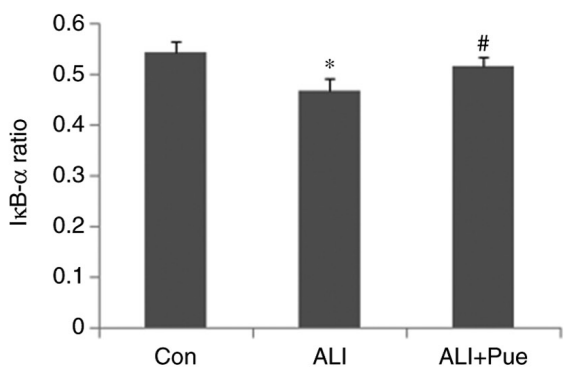

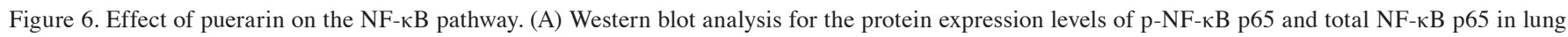
tissues. Results were quantified as the ratio of phosphorylated to total protein. (B) Western blot analysis for the protein expression levels of IкB- $\alpha$ in lung tissues. Results were quantified as the ratio of IкB- $\alpha$ signal to GAPDH. Data are presented as mean \pm standard error of the mean from three independent

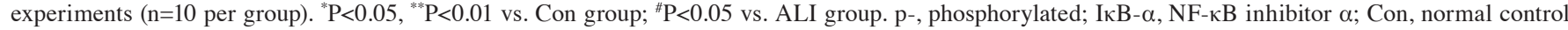
group; ALI, smoke inhalation group; ALI + Pue, puerarin treatment plus smoke inhalation group.

fluids (14). However, recent studies have suggested that activation of the local RAS in the lungs may contribute to the pathogenesis of ALI/ARDS via regulation of inflammation, vascular permeability and fibroblast activity (15). As a proinflammatory mediator, Ang II is reported to influence the inflammatory process (16-18). Ang II is involved in the progression of lung injury via several mechanisms, including activating inflammation in the lung, exerting a proapoptotic effect on alveolar epithelial cells and promoting fibrosis in ALI (19). The present study revealed that puerarin significantly decreased Ang II levels locally in the lung. However, the levels of Ang II in the circulation were not notably reduced by puerarin. This result suggested that the local RAS may have a pathogenic role in the lungs, independently of the RAS in the circulation. Ang II can promote inflammatory responses, including mononuclear cell proliferation and chemotaxis, and the recruitment of proinflammatory cells to the site of injury. Additionally, inflammatory cells can produce Ang II, thus contributing to the perpetuation of tissue damage (18). Ang II can induce IL- 6 production, which together with TGF- $\beta$ 


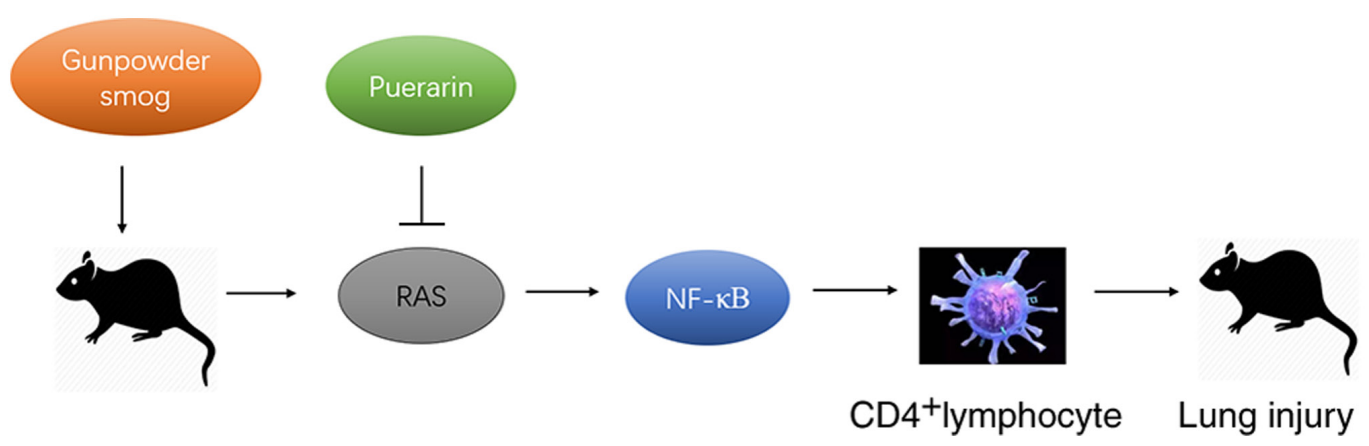

Figure 7. Schematic diagram of the protective mechanisms of puerarin in gunpowder smog-induced acute lung injury. RAS, renin-angiotensin system.

can promote the differentiation of IL-17A-producing Th17 cells $(8,9)$. Accordingly, the present study demonstrated that puerarin reduced the concentration of inflammatory cytokines, including IL-6, IL-1 $\beta$, IL-17A and TNF- $\alpha$, and this may be attributed to the reduced levels of Ang II by puerarin.

The majority of the biological functions of Ang II are mediated by binding to its receptor, AT1-R (20). In the present study, puerarin suppressed the expression of AT1-R in lung tissue. In addition, it has previously been shown that the Ang-II/AT1-R pathway induces the activation of the NF- $\kappa \mathrm{B}$ pathway and contributes to apoptosis of alveolar epithelial cells in seawater inhalation-induced lung injury (21). p-NF- $\kappa \mathrm{B}$ p65 levels reflect the activation of $\mathrm{NF}-\kappa \mathrm{B}$ signaling. I $\mathrm{B}-\alpha$ is the primary subset of $\mathrm{I} \kappa \mathrm{B}$, and the major function of $I \kappa B-\alpha$ is to regulate activation of NF- $\kappa B$. In the present study, administration of puerarin downregulated smoke inhalation-induced expression of $\mathrm{p}-\mathrm{NF}-\kappa \mathrm{B}$ p 65 and upregulated the smoke inhalation-suppressed expression of I $\kappa-\alpha$, suggesting that puerarin may inhibit the activation of $\mathrm{NF}-\kappa \mathrm{B} . \mathrm{NF}-\kappa \mathrm{B}$ pathways are involved in the regulation of proinflammatory cytokines, such as IL-6 and TNF- $\alpha$ (22). Suppression of the NF- $\mathrm{NB}$ pathway is consistent with the reduced levels of IL- 6 , IL-1 $\beta$ and TNF- $\alpha$ following treatment with puerarin.

The lungs are the major organs that express Ang II and ACE in humans and mice. The expression of ACE/Ang-II is markedly elevated in patients with ARDS (23). ACE has been reported to be upregulated in ARDS, and this is induced by pneumonia, trauma, aspiration, and pancreatitis (24-26). These previous studies demonstrated that ACE, Ang II and AT1-R promoted disease pathogenesis, induced lung edema and impaired lung function. In addition, mice deficient for ACE exhibited significant improvement in the symptoms. Furthermore, studies using ACE2 knockout mice have demonstrated that ACE2 protects murine lungs from ARDS (14). The negative regulation of Ang II accounts, in part, for the protective functions of ACE2 in ARDS. In the present study, puerarin reduced the expression of ACE, whilst enhancing the levels of ACE2. These results were consistent with the decreased levels of Ang II following puerarin treatment. Therefore, puerarin may attenuate inflammatory responses in gunpowder smog-induced ALI, at least in part by upregulating the expression of ACE2 and downregulating that of ACE, Ang II and AT1-R.

Cumulatively, the present study is the first to report that the possible immunoregulative mechanisms of puerarin in gunpowder smog-induced ALI may partly be attributed to inhibition of expression of Ang II, AT1-R and ACE and promotion of ACE2. However, as results in rats do not always translate in humans, future studies should confirm these findings in samples from patients with smoke inhalation-induced acute lung injury. Additionally, larger animal cohorts in vivo and multiple cell lines in vitro may be beneficial to further confirm the related mechanisms.

In conclusion, puerarin treatment suppressed the smoke inhalation-induced expression of Ang II, AT1-R and ACE, whilst enhancing the smoke inhalation-suppressed expression of ACE2, together with the downregulation of NFKB. Therefore, puerarin treatment significantly ameliorated the inflammation in gunpowder smog-induced ALI, at least partly by regulating the RAS and $N F-\kappa B$ pathways (Fig. 7). The present findings provided evidence for its potential application in the clinical management of smoke inhalation-induced acute lung injury that accounts for extensive morbidity and mortality.

\section{Acknowledgements}

Not applicable.

\section{Funding}

The present study was supported by the National Natural Scientific Foundation of China (grant no. 81700077), and the Military Medical Scientific Research Project (grant no. CWS12J021).

\section{Availability of data and materials}

The datasets used and/or analyzed during the present study are available from the corresponding author on reasonable request.

\section{Authors' contributions}

FZ designed the project, wrote the manuscript and performed the immunohistochemistry experiments. YW performed the western blot analysis. PL and PD performed the measurement of arterial blood gases. ML performed the cytokine assays. $\mathrm{CW}$ was responsible for the design of the project, the revision of the manuscript and performed some of the experiments. ML and $\mathrm{CW}$ are responsible for confirming the authenticity of the raw data. All authors read and approved the final manuscript. 


\section{Ethics approval and consent to participate}

All experimental procedures were approved by the Ethics Committee of the Medical College of the People's Liberation Army [approval no. SCXK (Beijing) 2012-0001].

\section{Patient consent for publication}

Not applicable.

\section{Competing interests}

The authors declare that they have no competing interests.

\section{References}

1. Fukuda S, Niimi Y, Andersen CR, Manyeza ER, Rojas JD, Prough DS and Enkhbaatar P: Blood carboxyhemoglobin elimination curve, half-lifetime, and arterial-venous differences in acute phase of carbon monoxide poisoning in ovine smoke inhalation injury model. Biochem Biophys Res Commun 526: $141-146,2020$

2. Mokra D and Kosutova P: Biomarkers in acute lung injury. Respir Physiol Neurobiol 209: 52-58, 2015.

3. Zhang F, Li MY, Lan YT and Wang CB: Imbalance of Th17/Tregs in rats with smoke inhalation-induced acute lung injury. Sci Rep 6: 21348, 2016.

4. Zhang F, Wang Z, Li M, Lan Y, Chen Y and Wang C: Puerarin attenuates smoke inhalation injury by regulation of Th1/Th2 expression and inhibition of Th17 cells in rats. Int Immunopharmacol 28: 546-553, 2015.

5. Tan WS, Liao W, Zhou S, Mei D and Wong WF: Targeting the renin-angiotensin system as novel therapeutic strategy for pulmonary diseases. Curr Opin Pharmacol 40: 9-17, 2018.

6. Platten M, Youssef S, Hur EM, Ho PP, Han MH, Lanz TV, Phillips LK, Goldstein MK, Bhat R, Raine CS, et al: Blocking angiotensin-converting enzyme induces potent regulatory $\mathrm{T}$ cells and modulates TH1- and TH17-mediated autoimmunity. Proc Nat Acad Sci U S A 106: 14948-14953, 2009.

7. He L, Du J, Chen Y,Liu C,Zhou M, Adhikari S, Rubin DT, Pekow J and Li YC: Renin-angiotensin system promotes colonic inflammation by inducing TH17 activation via JAK2/STAT pathway. Am J Physiol Gastrointest Liver Physiol 316: G774-G784, 2019.

8. Ju X, Ijaz T, Sun H, Ray S, Lejeune W, Lee C, Recinos A 3rd, Guo DC, Milewicz DM, Tilton RG and Brasier AR: Interleukin-6-signal transducer and activator of transcription-3 signaling mediates aortic dissections induced by angiotensin II via the T-helper lymphocyte 17-interleukin 17 axis in C57BL/6 mice. Arterioscler Thromb Vasc Biol 33: 1612-1621, 2013.

9. Bettelli E, Carrier Y, Gao W, Korn T, Strom TB, Oukka M, Weiner HL and Kuchroo VK: Reciprocal developmental pathways for the generation of pathogenic effector TH17 and regulatory T cells. Nature 441: 235-238, 2006.

10. Yue J, Chang SW, Xiao ZX, Qi YF and He JX: The protective effect of puerarin on angiotensin II-induced aortic aneurysm formation by the inhibition of NADPH oxidase activation and oxidative stress-triggered AP-1 signaling pathways. Oncol Lett 16: 3327-3332, 2018.

11. Chen G, Pan SF, Cui XL and Liu LH: Puerarin attenuates angiotensin II-induced cardiac fibroblast proliferation via the promotion of catalase activity and the inhibition of hydrogen peroxide-dependent Rac-1 activation. Chin J Nat Med 16: 41-52, 2018.
12. Tipnis SR, Hooper NM, Hyde R, Karran E, Christie G and Turner AJ: A human homolog of angiotensin-converting enzyme. Cloning and functional expression as a captopril-insensitive carboxypeptidase. J Biol Chem 275: 33238-33243, 2000.

13. Bai S, Huang ZG, Chen L, Wang JT and Ding BP: Effects of felodipine combined with puerarin on ACE2-Ang (1-7)-Mas axis in renovascular hypertensive rat. Regul Pept 184: 54-61, 2013.

14. Imai Y, Kuba K and Penninger JM: The discovery of angiotensin-converting enzyme 2 and its role in acute lung injury in mice. Exp Physiol 93: 543-548, 2008.

15. Marshall RP, Webb S, Bellingan GJ, Montgomery HE, Chaudhari B, McAnulty RJ, Humphries SE, Hill MR and Laurent GJ: Angiotensin converting enzyme insertion/deletion polymorphism is associated with susceptibility and outcome in acute respiratory distress syndrome. Am J Respir Crit Care Med 166: 646-650, 2002.

16. Miyoshi M, Nagata K, Imoto T, Goto O, Ishida A and Watanabe T: ANG II is involved in the LPS-induced production of proinflammatory cytokines in dehydrated rats. Am J Physiol Regul Integr Comp Physiol 284: R1092-R1097, 2003.

17. Schieffer B, Schieffer E, Hilfiker-Kleiner D, Hilfiker A, Kovanen PT, Kaartinen M, Nussberger J, Harringer W and Drexler H: Expression of angiotensin II and interleukin 6 in human coronary atherosclerotic plaques: Potential implications for inflammation and plaque instability. Circulation 101: 1372-1378, 2000.

18. Ruiz-Ortega M, Lorenzo O, Suzuki Y, Rupérez M and Egido J: Proinflammatory actions of angiotensins. Curr Opin Nephrol Hypertens 10: 321-329, 2001.

19. Marshall RP, Puddicombe A, Cookson WO and Laurent GJ: Adult familial cryptogenic fibrosing alveolitis in the United Kingdom. Thorax 55: 143-146, 2000.

20. Wang D, Chai XQ, Magnussen CG, Zosky GR, Shu SH, Wei X and Hu SS: Renin-angiotensin-system, a potential pharmacological candidate, in acute respiratory distress syndrome during mechanical ventilation. Pulm Pharmacol Ther 58: 101833, 2019.

21. Wang Y, Zhu Y, Zhu Y, Lu Z and Xu F: Regulation of the angiotensin II-p22phox-reactive oxygen species signaling pathway, apoptosis and 8-oxoguanine-DNA glycosylase 1 retrieval in hyperoxia-induced lung injury and fibrosis in rats. Exp Ther Med 13: 3397-3407, 2017.

22. Li Q and Verma IM: NF-kappaB regulation in the immune system. Nat Rev Immunol 2: 725-734, 2002.

23. Wenz M, Steinau R, Gerlach H, Lange M and Kaczmarczyk G: Inhaled nitric oxide does not change transpulmonary angiotensin II formation in patients with acute respiratory distress syndrome. Chest 112: 478-483, 1997.

24. Imai Y, Kuba K, Rao S, Huan Y, Guo F, Guan B, Yang P, Sarao R, Wada T, Leong-Poi H, et al: Angiotensin-converting enzyme 2 protects from severe acute lung failure. Nature 436: 112-116, 2005.

25. Zou Z, Yan Y, Shu Y, Gao R, Sun Y, Li X, Ju X, Liang Z, Liu Q, Zhao Y, et al: Angiotensin-converting enzyme 2 protects from lethal avian influenza A H5N1 infections. Nat Commun 5: 3594, 2014.

26. Podowski M, Calvi C, Metzger S, Misono K, Poonyagariyagorn $\mathrm{H}$, Lopez-Mercado A, Ku T, Lauer T, McGrath-Morrow S, Berger A, et al: Angiotensin receptor blockade attenuates cigarette smoke-induced lung injury and rescues lung architecture in mice. J Clin Invest 122: 229-240, 2012.

This work is licensed under a Creative Commons Attribution-NonCommercial-NoDerivatives 4.0 International (CC BY-NC-ND 4.0) License. 